\title{
Infección natural de Panstrongylus rufotuberculatus con Trypanosoma cruzi (Kinetoplastida: Trypanosomatidae) en Costa Rica
}

\author{
OLGER CALDERÓN ARGUEDAS*, ADRIANA TROYO* y MISAEL CHINCHILLA****
}

\author{
Trypanosoma cruzi (KINETOPLASTIDA: TRYPANOSOMATIDAE) NATURAL INFECTION IN \\ Panstrongylus rufotuberculatus FROM COSTA RICA
}

An adult female of Panstrongylus rufotuberculatus was collected in "Manuel Antonio" National Park (Puntarenas, Costa Rica). The microscopic analysis of the insect intestinal content showed the presence of Trypanosoma cruzi epimastigote and metacyclic triposmatigote forms. The characteristic T. cruzi blood tripomastigotes were observed in $\mathrm{C}_{3} \mathrm{H}$ mice 12 days after their intra peritoneal inoculation with the insect gut material. This is the first report in the biomedical literature of Costa Rica about the presence of $\boldsymbol{T}$. cruzi in this Chagas' disease vector.

Key words: Pantrongylus rufotuberculatus, Reduviid, Triatominae, Trypanosoma cruzi, Chagas ‘ disease, Costa Rica.

\section{INTRODUCCIÓN}

Panstrongylus rufotuberculatus es la segunda especie de Panstrongylus más abundante en Costa Rica, solamente superada por $P$. geniculatus ${ }^{1}$.

Su presencia en Costa Rica se conoce desde mediados de la década del 50 cuando se informó el hallazgo del triatomino en la localidad de La Fortuna (Burío), cantón de San Carlos, Provincia de Alajuela. En esta ocasión, el examen del contenido intestinal mostró resultados negativos por la presencia de $T$. cruzi $i^{2}$.

El presente informe es el primero relativo a Costa Rica en que se informa acerca de la infección con $T$. cruzi en esta especie de vector, lo que de alguna manera sugiere el eventual papel que podría mostrar el insecto en la transmisión de la enfermedad de Chagas.

\section{MATERIAL Y MÉTODOS}

Una hembra adulta de $P$. rufotuberculatus fue colectada en el Parque Nacional "Manuel Antonio", en la costa pacífica de Costa Rica (Cantón Quepos, Provincia de Puntarenas). Dicho ejemplar fue transportado al laboratorio donde se realizó su correspondiente identificación taxonómica siguiendo una clave taxonómica ${ }^{3}$. Luego, se realizó su disección y una muestra de contenido intestinal fue emulsificada en solución

\footnotetext{
Centro de Investigación en Enfermedades Tropicales (CIET). Departamento de Parasitología, Facultad de Microbiología, Universidad de Costa Rica.

** Laboratorio de Investigación. Universidad de Ciencias Médicas “Dr. Andrés Vesalio Guzmán”. San José, Costa Rica.
} 
salina al $0,85 \%$ para su posterior observación microscópica. $0,5 \mathrm{ml}$ de esta suspensión fue inoculada por vía intraperitoneal en ratones $\mathrm{C}_{3} \mathrm{H}$ de $20 \mathrm{~g}$. Adicionalmente se realizó una suspensión de dicho material en suero humano, la cual fue extendida en un portaobjetos para su posterior fijación y tinción con colorante de Giemsa. La observación de la tinción se realizó por medio del microscopio de luz utilizando el objetivo de inmersión (100X).

La revisión bibliográfica se hizo mediante la consulta de las siguientes fuentes: "Primera bibliografía anotada sobre enfermedad de Chagas en Costa Rica (1895-1982)"4, "Indice de Publicaciones entomológicas de Costa Rica" y MEDLINE/Pub Med ${ }^{6}$ en Internet.

\section{RESULTADOS}

La identificación taxonómica del ejemplar colectado se ajusta a las características apuntadas para $P$. rufotuberculatus ${ }^{3}$ (Figura 1). El análisis de los frotis fecales mostró las formas flageladas típicas (epimastigotos y tripomastigotos metacíclicos) de T. cruzi. La presencia de T. cruzi

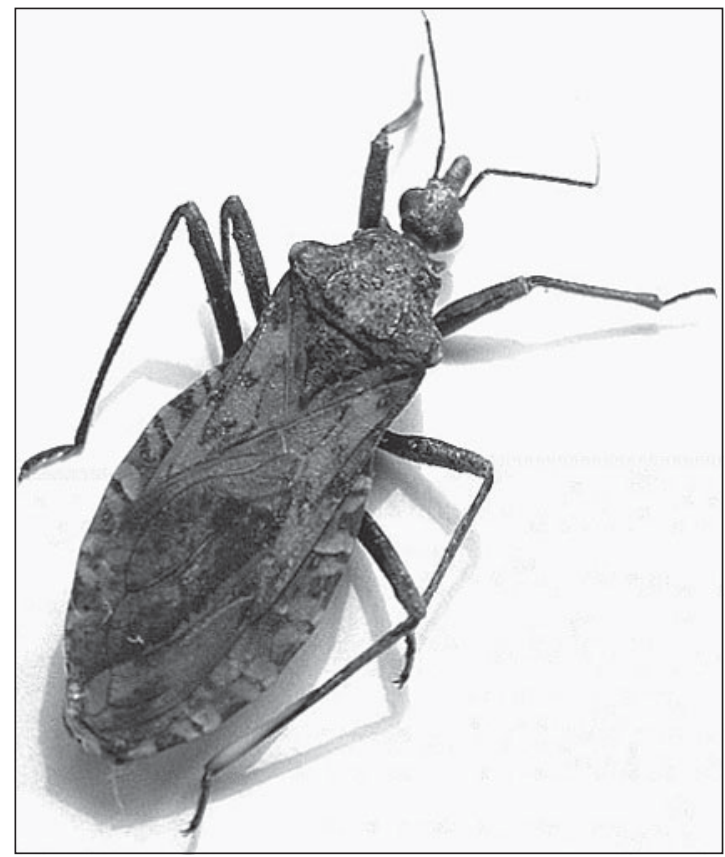

Figura 1. Panstrongylus rufotuberculatus positivo por Trypanosoma cruzi colectado en el Parque Nacional. "Manuel Antonio", Puntarenas, Costa Rica. en el torrente sanguíneo de los ratones se pudo corroborar luego de 12 días de su inoculación.

No se pudo evidenciar la existencia de ningún informe en la literatura biomédica costarricense acerca de la infección de $P$. rufotuberculatus con T. cruzi.

\section{DISCUSIÓN}

La presencia de $P$. rufotuberculatus, como uno de los reduviideos presentes en Costa Rica, ha sido documentada ${ }^{1,2,3,7}$.

Estudios realizados de la colección de triatominos del Instituto Nacional de Biodiversidad, la cual fue elaborada con ejemplares colectados en trampas de luz, muestran que $P$. rufotuberculatus es el cuarto triatomino en orden de frecuencia de colecta, solamente superado por Triatoma dimidiata, $T$. dispar y $P$. geniculatus ${ }^{1}$.

El presente hallazgo de $P$. rufotuberculatus en la costa pacífica de Costa Rica, concuerda con lo que se ha demostrado para esta especie, la cual es frecuente en regiones con altitudes menores de 800 metros sobre el nivel del mar $^{1}$. La ocurrencia de infección por $T$. cruzi en el triatomino demuestra que éste está cumpliendo un papel vectorial en el ambiente circundante al sitio colecta. A pesar de que $P$. rufotuberculatus se ha relacionado con animales salvajes como procyonidos, murciélagos y marsupiales ${ }^{1}$, en algunos países se ha demostrado una marcada tendencia hacia la domiciliación. En estudios realizados en casas de una comunidad de La Gardenia, Colombia, se pudo evidenciar la presencia de 94 formas evolutivas de $P$. rufotuberculatus (2 huevos no eclosionados, 81 ninfas, 10 adultos y un cuerpo incompleto), en 3 de 40 casas $^{8}$. En este estudio, el $100 \%$ de las formas colectadas procedían del intradomicilio. El hecho de que en el estudio anteriormente citado la infección natural por T. cruzi fuera del $4,5 \%$ lo vincula como uno de los potenciales vectores de $T$. cruzi en ciertos ambientes rurales de Colombia. Las zonas aledañas al Parque Nacional de "Manuel Antonio" han experimentado en los últimos 20 años un marcado desarrollo urbanístico condicionado por el incremento en la actividad turística. La presencia de $P$. rufotuberculatus positivos por $T$. cruzi en esta zona, asociada con la tendencia a la domiciliación y la influencia de la luz eléctrica como atrayente, le confieren una potencial importancia en su papel vectorial 
implicando la posibilidad de poder transmitir el parásito al ser humano y animales domésticos.

\section{RESUMEN}

Un ejemplar hembra de T. rufotuberculatus fue colectado en el Parque Nacional "Manuel Antonio" (Puntarenas, Costa Rica). El análisis microscópico de su contenido intestinal mostró la presencia de formas de epimastigotos y tripomastigotos metacíclicos de $T$. cruzi. La presencia de tripomastigotos sanguíneos del parásito fue evidenciada en ratones $\mathrm{C}_{3} \mathrm{H} 12$ días luego de la inoculación intraperitoneal de los mismos con material fecal del insecto.

El presente trabajo es el primer informe en la literatura biomédica de Costa Rica sobre la presencia de $T$. cruzi en esta especie de vector de la enfermedad de Chagas.

\section{REFERENCIAS}

1-. ZELEDON R, UGALDE J A, PANIAGUA L. Entomological and ecological aspects of six sylvatic species of triatomines (Hemiptera, Reduviidae) from the collection of the National Biodiversity Institute of Costa Rica, Central America. Mem Inst Oswaldo Cruz 2001; 96: 757-64.

2.- ZELEDON R, VARGAS M. Panstrongylus rufotuberculatus (Champion, 1899) en Costa Rica y breve mención de las especies costarricenses de Triatominae (Hemiptera, Reduviidae). Rev Biol Trop 1955; 3: 179-82.

3.- LENT H, WYGODZINSKY P. Revision of the triatominae (Hemiptera, Reduviidae) and their significance as vectors of Chagas ' disease. Bull Am Museum Nat Histor 1979; 163: 125-520.

4.- VARGAS L, JIRON L. Primera bibliografía anotada sobre enfermedad de Chagas en Costa Rica (18951982). Caja Costarricense de Seguro Social. Publicaciones bibliográficas, serie 110. Número 001, San José Costa Rica, 1983. 22 pp.

5.- JIRON L, SANCHO DE BARQUERO M. Índice de publicaciones entomológicas de Costa Rica. Consejo Nacional de Investigaciones Científicas y Tecnológicas (CONICIT)/Organization of Tropical Studies (OTS)/ Universidad de Costa Rica (UCR). Primera Edición, San José Costa Rica. 1983. 305 pp.

6.- http://www.ncbi.nlm.nih.gov/entrez/query.fcgi

7.- COSCORON M C, JIRON L F. Updated checklist of assasin bug species (Hemiptera: Reduviidae) of Costa Rica. Brenesia 1988; 29: 107-13.

8.- WOLFF M, CASTILLO, D. Domiciliation trend of Panstrongylus rufotuberculatus in Colombia. Mem Inst Oswaldo Cruz 2002; 97: 297-300.

Agradecimientos: Los autores desean manifestar su agradecimiento al Dr. Gustavo Gutérrez Espeleta y a la Vicerrectoría de Investigación de la Universidad de Costa Rica por el aporte dado a través del proyecto VI 111-A1015 y al Dr. Dennis León Alán del Departamento de Parasitología de la Facultad de Microbiología en la Universidad de Costa Rica por la elaboración de la fotografía presentada. 